Giovana Sposito ${ }^{1}$

Maria José D'Elboux ${ }^{1}$

Anita Liberalesso Neri ${ }^{1}$

Maria Elena Guariento ${ }^{1}$

\section{A satisfação com a vida e a funcionalidade em idosos atendidos em um ambulatório de geriatria}

\author{
Satisfaction with life and functionality \\ among elderly patients in a geriatric outpatient clinic
}

${ }^{1}$ Departamento de Gerontologia, Faculdade de Ciências Médicas, Unicamp. R. Tessália Vieira de Camargo 126, Barão Geraldo. 13.083-887 Campinas SP Brasil giovanasposito@gmail.com
Abstract Aging is often accompanied by functional limitations that affect self esteem, lowering the level of satisfaction with life. Studies highlight satisfaction with life as a predictor of quality of life and has a marked correlation with a decrease in functionality. Therefore, this study sought to examine the relationship between satisfaction with life and functional independence and performance of the lower limbs (muscle strength, gait speed and balance) among the elderly in outpatient care with respect to age groups and genders. A total of 125 elderly men and women aged 60 years and above, attending a geriatric outpatient clinic. The instruments used were: 1) Functional Independence Measure (FIM) to evaluate functional dependence; 2) Short Physical Performance Battery (SPPB) to measure physical performance; 3) Satisfaction with life. The sample was random, with a predominance of females, in which the older participants had greater functional impairment. In univariate logistic regression analysis and multivariate analysis, overall self reported and comparative satisfaction with life was more satisfactory among the oldest elderly. The results suggest that older individuals have better satisfaction with life even though they have greater functional impairment.

Key words Functional capacity, Satisfaction with life, Elderly
Resumo O envelhecimento pode vir acompanhado de limitações funcionais que influenciam a autoestima, rebaixando o nível de satisfação com a vida. Estudos destacam a satisfação com a vida como preditor na qualidade de vida e tem forte relação com a queda da funcionalidade. Portanto, este estudo teve como objetivo verificar a relação entre a satisfação com a vida e a independência funcional e o desempenho de membros inferiores (força muscular, velocidade de marcha e equilíbrio) de idosos em seguimento ambulatorial, em relação a grupos etários e de gêneros. Foram avaliados 125 idosos de ambos os sexos com idade minima de 60 anos, atendidos em um ambulatório de geriatria. Os instrumentos utilizados foram: 1) Medida da Independência Funcional (MIF) para avaliar a dependência funcional; 2) Short Physical Performance Battery (SPPB) para medir o desempenho físico; 3) Satisfação com a vida. A amostra utilizada foi de conveniência, com predominio do sexo feminino, os idosos mais velhos apresentaram maior comprometimento funcional. Na análise de regressão logística univariada e multivariada, a satisfação global com a vida autorrelatada e comparada foi mais satisfatória entre os idosos mais velhos. Os resultados sugerem que individuos mais velhos apresentam melhor satisfação com a vida mesmo com maior comprometimento funcional.

Palavras-chave Capacidade funcional, Satisfação com a vida, Idoso 


\section{Introdução}

Com aumento da expectativa de vida e as possíveis modificações físicas, psicológicas e sociais causadas pelo envelhecimento, torna-se de fundamental importância propiciar uma boa qualidade de vida a essa população.

A qualidade de vida é a combinação de indicadores subjetivos, que são demonstrados por meio de sensações de relações afetivas como felicidade e satisfação, saúde percebida, autocontrole, estresses e competência social ${ }^{1}$.

Embora seja um conceito subjetivo, a qualidade de vida é influenciada por condições objetivas, tais como as condições de saúde, relações sociais, realização de atividades, entre outras, que influenciam diretamente a satisfação com viver ${ }^{2-4}$.

A satisfação com a vida (SV) não se refere somente à saúde física, mas também às necessidades de satisfação social e psicológica ${ }^{5}$. Está associada a gênero, idade, nível socioeconômico e educacional, entre outros. Além disso, é um forte indicador de qualidade de vida percebida e pode ser considerada um guia norteador para políticas de saúde na velhice ${ }^{6}$.

Estudos indicam que a SV é influenciada por estruturas do conhecimento de si mesmo, bem como um conjunto de funções cognitivas de várias áreas do funcionamento pessoal ${ }^{1}$. A avaliação da SV reflete as expressões de cada pessoa aos próprios critérios, ou seja, o modo e os motivos que levam as pessoas a viverem as experiências de maneira positiva ${ }^{7}$.

A descrição do julgamento cognitivo é um reflexo individual de cada pessoa baseados na combinação de saúde física e mental, papeis sociais e fatores ambientais associados à capacidade funcional. Ou seja, a diminuição da funcionalidade ocasionada pelo envelhecimento pode refletir diretamente na $\mathrm{SV}^{8,9}$.

Nos idosos, a percepção da vida em comparação com outras pessoas da mesma idade, estabelece o que e como eles sentem suas condições de vida, que emergem de discernimento próprio que varia de uma pessoa a outra ${ }^{10}$.

Entretanto, sabe-se que a SV e a satisfação com a vida quando comparada (SVC) podem ser influenciadas por variáveis de natureza física, como a independência funcional e a mobilidade física, o que torna o presente estudo relevante para identificar a associação entre a SV e os testes funcionais em idosos.

Portanto, o presente estudo tem como objetivo verificar as associações entre a satisfação com a vida e satisfação com a vida comparada com outros de mesma idade e a independência funcional e a mobilidade e a flexibilidade de idosos em seguimento ambulatorial considerando as variáveis de gênero e idade.

\section{Método}

Este estudo foi desenvolvido a partir do banco de dados do Projeto Temático intitulado, "Qualidade de vida em idosos: indicadores de fragilidade e de bem-estar subjetivo" o qual teve o objetivo de identificar e analisar os fatores de predição e de discriminação de fragilidade relacionados à saúde física e mental em idosos residentes no Município de Campinas e região, correlacionados ao impacto dessas variáveis sobre a qualidade de vida. $\mathrm{O}$ estudo foi realizado no ambulatório de geriatria do hospital universitário.

Os idosos foram convidados a participar da pesquisa ao comparecerem no ambulatório para atendimento e, em caso afirmativo, assinavam o Termo de Consentimento Livre e Esclarecido conforme recomendação. Em caso de recusa não houve prejuízo do seu atendimento e tratamento no serviço.

Para a coleta de dados foi desenvolvido, testado e utilizado um protocolo composto por 17 seções quem incluíam questões fechadas e escalas de avaliação de reconhecimento científico e clínico no âmbito da gerontologia, sobre condições de vida, saúde física e mental, avaliação funcional, medida de desempenho físico e bem-estar subjetivo.

O estudo foi aprovado pelo Comitê de Ética da Faculdade de Ciências Médicas da Universidade Estadual de Campinas, conforme Resolução 196/96 ${ }^{11}$.

\section{Sujeitos}

Foi selecionada uma amostra de 125 idosos, de ambos os sexos, com idade mínima de 60 anos, em condições físicas e mentais de compreender e responder ao protocolo e que concordaram em participar do estudo, de acordo com o Consentimento Livre e Esclarecido. Foram excluídos da pesquisa, idosos com dificuldades de comunicação e expressão para responder aos instrumentos e aqueles que se recusaram a participar do estudo.

\section{Coleta de dados}

Para o presente estudo foram utilizadas as seguintes variáveis 
- Sexo e faixa etária, graduada em três níveis: $60-69$ anos, $70-79$ anos e 80 anos ou mais.

- Avaliação da satisfação com a vida ${ }^{12}$. Foram avaliados os seguintes itens: duas questões sobre satisfação global, a primeira sobre a satisfação do idoso com a própria vida e a outra, sobre a satisfação com a vida quando comparada à outra pessoa da mesma idade, com o escore de 1 ponto para as respostas pouco, 2 pontos para as respostas mais ou menos e 3 pontos para as respostas muito.

- Mobilidade e Flexibilidade: avaliado por meio do instrumento Short Phisical Performance Battery (SPPB $)^{13}$, composto por três testes que avaliam o equilíbrio, a marcha e a força de membros inferiores. O equilíbrio é avaliado em três posições dos pés; em paralelo, com o hálux encostado tanto na borda medial como na posterior do calcanhar. Foi atribuído 1 ponto se realizado em tempo $\leq$ que 10 segundos e 0 (zero) se for $\geq$ a 10 segundos, para os dois primeiros testes. No terceiro teste a pontuação varia de 0 (zero) para $<3$ segundos, 1 entre 3 a 9.99 segundos e 2 se for $\geq$ a 10 segundos. Para a avaliação da marcha utilizou-se um cronometro para registrar o tempo que o indivíduo levou para percorrer um corredor de quatro metros com o tempo de ida e volta, repetindo duas vezes o percurso. A pontuação pôde variar de: o (zero) quando incapaz, 1 se $>$ que 8.70 segundos, 2 entre 6.21 e 8.7 segundos, 3 entre 4.82 e 6.20 segundos e 4 se for $<$ que 4.82 segundos. A força muscular dos membros inferiores é verificada através da velocidade que o idoso precisa para se levantar de uma cadeira com os membros superiores cruzados sobre o peito, repetindo o teste 5 vezes consecutivas. A pontuação varia de acordo com o tempo gasto: 0 (zero) quando incapaz, 1 se > 16.7, 2 entre 13.70 e 16.69 segundos, 3 entre 11.20 e 13.69 segundos e 4 se $<$ a 11.19 segundos. O escore total do SPPB, obtido pela soma da pontuação de cada teste que varia de 0 (zero) a 12 pontos, representa o desempenho dos membros inferiores dos idosos através da seguinte graduação: 0 (zero) a 3 pontos quando é incapaz ou mostra desempenho muito ruim, 4 a 6 pontos representa baixo desempenho, 7 a 9 pontos em caso de moderado desempenho e 10 a 12 pontos ao apresentar bom desempenho.

- Medida da Independência Funcional $(\mathrm{MIF})^{14}$ : Avaliação da necessidade de ajuda de outra pessoa nas atividades de vida diária. Contêm 18 itens que avaliam o quanto o idoso é independente/dependente para a prática de suas atividades diárias como cuidados pessoais, controle de esfíncteres, mobilidade e transferência, locomoção e cognição social. A pontuação varia de 1 (dependência total) a 7 pontos (independência total) para cada tarefa, sendo a variação total da MIF de 18 a 126 pontos.

\section{Analise dos dados}

Os dados foram submetidos às seguintes análises:

- Descritiva, com medidas de posição (média, mediana, mínima e máxima) e dispersão (desvio-padrão), para o tratamento dos dados sociodemográficos e escores dos instrumentos utilizados.

- Analise de regressão logística, realizada a análise univariada e multivariada ${ }^{15}$. O critério de seleção de variáveis foi o Stepwise ou passo a passo, que considera todas, seja ou não significativa na analise univariada e, em seguida, são selecionadas apenas aquelas que são mais significativas conjuntamente, ao nível de significância de 5\% $(\mathrm{p}<0,05)$ para o modelo final da análise multivariada.

A SV e a SVC foram analisados como variáveis dependentes, enquanto sexo, faixa etária, desempenho físico (escores do SPPB) e independência funcional (escores da MIF) foram as variáveis independentes.

- Confiabilidade, com determinação do coeficiente Alpha de Cronbach, para avaliar a consistência interna dos instrumentos, valor considerado satisfatório $>0,70$.

O nível de significância adotado para os testes estatísticos foi de 5\% ( $\mathrm{p}<0,05)$.

\section{Resultados}

Dos 125 idosos participantes deste estudo, 61,6\% são mulheres; a idade variou entre 60 a 93 anos, sendo desses $44 \%$ com idade entre 70 a 79 anos e $36,8 \%$ com 80 anos ou mais, conforme descrito na Tabela 1. Observa-se também, melhores resultados da SPPB nos homens e em idosos de 70 a 79 anos. Já a SVC identificou melhores resultados em idosos mais velhos e nas mulheres.

Na Tabela 2, podemos observar a média da pontuação do SBBP Total para os homens foi de $6,67( \pm 2,08)$. Já a pontuação média MIF Total foi de $110,0( \pm 14,02)$ para as mulheres e 117,6 $( \pm$ 9,17 ) para os homens, revelando independência funcional desses idosos. A média da SV nos idosos entre 60 a 69 anos foi de 1,96 $( \pm 0,75)$ e a média da SVC entre os idosos com 80 anos ou mais foi de 2,68 $( \pm 0,52)$. 
Tabela 1. Descrição da variação observada dos valores da SPPB Total, MIF Total, Satisfação com a vida e Satisfação com a vida comparada, conforme gênero e idade $(n=125)$. Campinas (SP).

\begin{tabular}{|c|c|c|c|c|c|}
\hline \multirow{2}{*}{$\begin{array}{c}\text { Variação } \\
\text { observada }\end{array}$} & \multicolumn{2}{|c|}{ Gênero } & \multicolumn{3}{|c|}{ Idade } \\
\hline & Masculino & Feminino & 60-69 anos & $70-79$ anos & $\geq 80$ anos \\
\hline & $48(38,40)$ & $77(61,60)$ & $24(19,20)$ & $55(44,00)$ & $46(36,80)$ \\
\hline \multicolumn{6}{|l|}{ SPPB Total } \\
\hline $0-3$ & $6(12,50)$ & $27(35,06)$ & $4(16,67)$ & $9(16,36)$ & $20(43,48)$ \\
\hline $4-6$ & $11(22,92)$ & $27(35,06)$ & $4(16,67)$ & $20(36,36)$ & $14(30,43)$ \\
\hline $7-9$ & $29(60,42)$ & $21(27,27)$ & $14(59,33)$ & $25(45,45)$ & $11(23,91)$ \\
\hline $10-12$ & $2(4,17)$ & $2(2,60)$ & $2(8,33)$ & $1(1,82)$ & $1(2,17)$ \\
\hline \multicolumn{6}{|l|}{ MIF Total } \\
\hline$<116$ & $14(29,17)$ & $43(57,33)$ & $11(45,83)$ & $22(40,00)$ & $24(54,55)$ \\
\hline$\geq 116$ & $34(70,83)$ & $32(42,67)$ & $13(54,17)$ & $33(60,00)$ & $20(45,45)$ \\
\hline \multicolumn{6}{|l|}{ SV } \\
\hline Pouco & $4(8,33)$ & $11(14,67)$ & $7(29,17)$ & $6(10,91)$ & $2(4,55)$ \\
\hline Mais ou menos & $17(35,42)$ & $31(41,31)$ & $11(45,83)$ & $22(40,00)$ & $15(34,09)$ \\
\hline Muito & $27(56,25)$ & $33(44,00)$ & $6(25,00)$ & $27(49,09)$ & $27(61,36)$ \\
\hline \multicolumn{6}{|l|}{ SVC } \\
\hline Pouco & $2(4,17)$ & $10(13,33)$ & $6(25,00)$ & $5(9,09)$ & $1(2,27)$ \\
\hline Mais ou menos & $16(33,33)$ & $28(37,33)$ & $8(33,33)$ & $24(43,64)$ & $12(27,27)$ \\
\hline Muito & $30(62,50)$ & $37(49,33)$ & $10(41,67)$ & $26(47,27)$ & $31(70,45)$ \\
\hline
\end{tabular}

$\mathrm{n}=$ número de sujeitos; $\mathrm{SPPB}=$ Short Phisical Performance Battery; MIF = Medida da Independência Funcional; SV = Satisfação com a vida; $S V C=$ Satisfação com a vida comparada.

Tabela 2. Análise comparativa das médias dos escores do SPPB Total, MIF Total, Satisfação com a vida e Satisfação com a vida comparada, conforme gênero e idade $(n=125)$. Campinas $(S P)$.

\begin{tabular}{|c|c|c|c|c|c|c|c|c|}
\hline & \multicolumn{2}{|c|}{ SPPB Total } & \multicolumn{2}{|l|}{ MIF Total } & \multicolumn{2}{|l|}{$\mathbf{S V}^{* *}$} & \multicolumn{2}{|l|}{ SVC $^{* *}$} \\
\hline & Média $\left( \pm d p^{*}\right)$ & $\mathbf{P}^{* * *}$ & Média $\left( \pm d p^{*}\right)$ & $\mathbf{P}^{* * *}$ & Média $\left( \pm d p^{*}\right)$ & $\mathbf{P}^{* * *}$ & Média $\left( \pm d p^{*}\right)$ & $\mathbf{P}^{* * *}$ \\
\hline Gênero & & $<0,001$ & & $<0,001$ & & 0,105 & & 0,092 \\
\hline Homens & $6,67( \pm 2,08)$ & & $117,6( \pm 9,17)$ & & $2,48( \pm 0,65)$ & & $2,58( \pm 0,58)$ & \\
\hline Mulheres & $4,82( \pm 2,59)$ & & $110,0( \pm 14,02)$ & & $2,29( \pm 0,71)$ & & $2,36( \pm 0,71)$ & \\
\hline Idade & & 0,003 & & 0,274 & & 0,004 & & 0,010 \\
\hline 60-60 anos & $6,29( \pm 3,01)$ & & $113,1( \pm 14,43)$ & & $1,96( \pm 0,75)$ & & $2,17( \pm 0,82)$ & \\
\hline 70-79 anos & $6,04( \pm 2,02)$ & & $115,1( \pm 10,22)$ & & $2,38( \pm 0,68)$ & & $2,38( \pm 0,65)$ & \\
\hline$\geq 80$ anos & $4,52( \pm 2,62)$ & & $110,1( \pm 14,58)$ & & $2,57( \pm 0,59)$ & & $2,68( \pm 0,52)$ & \\
\hline
\end{tabular}

" dp = Desvio padrão, ${ }^{* *}$ Missing $=2,{ }^{* * *} \mathrm{p}=$ Nível de significância. SPPB = Short Phisical Performance Battery; MIF = Medida da Independência Funcional; $\mathrm{SV}=$ Satisfação com a vida; $\mathrm{SVC}=$ Satisfação com a vida comparada.

$\mathrm{Na}$ análise de regressão logística univariada da SV e SVC com as variáveis gênero, idade, SPPB Total e MIF Total, verifica-se que apenas a idade 80 anos ou mais tiveram relevância estatística, conforme a Tabela 3. Os idosos com essa idade apresentaram 4,77 e 3,34 mais chances de relatarem satisfação autorreferida e comparada melhores do que os idosos entre 60 e 69 anos de idade.

Embora a análise univariada mostrar que a idade influenciou a SV autorreferida e a SVC, utilizou-se a análise multivariada para confirmar se esta variável mantém poder estatístico. $\mathrm{Na}$ análise multivariada da SV e SVC, observa-se na Tabela 4, que o avançar da idade aumenta as chances do idoso ser mais satisfeito com a vida. Idosos com 80 anos ou mais apresentaram 4,77 mais chances de melhor SV e 3,34 mais chances de melhor SV quando comparada.

Para a análise da consistência interna dos instrumentos utilizados foi realizado Alfa de Cron- 
Tabela 3. Análise de regressão logística univariada para SV e SVC $(n=125)$. Campinas (SP)

\begin{tabular}{|c|c|c|c|}
\hline & Valor-p & $\mathbf{O R}^{* *}$ & IC $95 \%$ OR \\
\hline \multicolumn{4}{|l|}{ SV } \\
\hline \multicolumn{4}{|l|}{ Gênero } \\
\hline Mulheres & & 1,00 & - - \\
\hline Homens & 0,186 & 1,64 & $0,79-3,40$ \\
\hline \multicolumn{4}{|l|}{ Idade } \\
\hline 60-69 anos & & 1,00 & -- \\
\hline 70-79anos & 0,051 & 2,89 & $0,99-8,39$ \\
\hline$\geq 80$ anos & 0,006 & 4,77 & $1,58-14,39$ \\
\hline SPPB Total & & & -- \\
\hline $0-3$ & & 1,00 & $0,66-4,48$ \\
\hline $4-6$ & 0,267 & 1,72 & $0,60-3,54$ \\
\hline $7-12$ & 0,400 & 1,46 & \\
\hline \multicolumn{4}{|l|}{ MIF Total } \\
\hline$<116$ & & 1,00 & -- \\
\hline$\geq 116$ & 0,666 & 0,86 & $0,42-1,74$ \\
\hline \multicolumn{4}{|l|}{ SVC } \\
\hline \multicolumn{4}{|l|}{ Gênero } \\
\hline Mulheres & & 1,00 & -- \\
\hline Homens & 0,154 & 1,71 & $0,82-3,59$ \\
\hline \multicolumn{4}{|l|}{ Idade } \\
\hline 60-69 anos & & 1,00 & -- \\
\hline 70-79anos & 0,646 & 1,26 & $0,48-3,31$ \\
\hline$\geq 80$ anos & 0,023 & 3,34 & $1,18-9,43$ \\
\hline \multicolumn{4}{|l|}{ SPPB Total } \\
\hline $0-3$ & & 1,00 & -- \\
\hline $4-6$ & 0,575 & 0,76 & $0,30-1,97$ \\
\hline 7-12 & 0,179 & 1,84 & $0,76-4,49$ \\
\hline \multicolumn{4}{|l|}{ MIF Total } \\
\hline$<116$ & & 1,00 & —- \\
\hline$\geq 116$ & 0,269 & 1,50 & $0,73-3,06$ \\
\hline
\end{tabular}

* Critério Stepwise de seleção de variáveis para análise de regressão logística univariada. SV = Satisfação com a vida; SVC $=$ Satisfação com a vida comparada. ${ }^{*}$ OR-Razão de chance para melhorar a SV e SVC, IC95\% OR-Intervalo de 95\% de confiança para OR.

bach com valor considerado satisfatório $>0,70$. Desta forma, todos os instrumentos utilizados no presente estudo apresentaram valores considerados consistentes.

\section{Discussão}

O estudo analisou a satisfação com a vida e sua relação com a independência funcional de idosos em acompanhamento ambulatorial, estudados comparados por meio de instrumentos específicos respondidos pelo próprio sujeito ou por seu acompanhante e teste de observação direta da mobilidade física.
Tabela 4. Análise de regressão logística multivariada para SV e SVC $(n=125)$. Campinas (SP)

\begin{tabular}{lccc}
\hline $\begin{array}{c}\text { Variáveis } \\
\text { selecionadas }\end{array}$ & Valor-p & OR $^{* *}$ & IC 95\% OR \\
\hline SV & & & \\
$\quad$ Idade & & & \\
$\quad$ 60-69 anos & & 1,00 & -- \\
$\quad$ 70-79anos & 0,051 & 2,89 & $0,99-8,39$ \\
$\quad \geq 80$ anos & $\mathbf{0 , 0 0 6}$ & 4,77 & $1,58-14,39$ \\
SVC & & & \\
Idade & & & \\
$\quad$ 60-69 anos & & 1,00 & -- \\
$\quad$ 70-79anos & 0,646 & 1,26 & $0,48-3,31$ \\
$\quad \geq 80$ anos & $\mathbf{0 , 0 2 3}$ & 3,34 & $1,18-9,43$ \\
& & & \\
\hline
\end{tabular}

${ }^{*}$ Critério Stepwise de seleção de variáveis para análise de regressão logística multivariada. $S V=$ Satisfação com a vida; SVC=Satisfação com a vida comparada. ${ }^{* *}$ OR-Razão de chance para melhorar a SV e SVC, IC95\% OR-Intervalo de 95\% de confiança para OR.

Assim como em outras pesquisas na área ${ }^{16-18}$ sobre gerontologia, a amostra contou com o predomínio do sexo feminino $(61,6 \%)$ e diminuição da funcionalidade entre idosos com idades mais avançadas (> 80 anos).

Corroborando com outras pesquisas ${ }^{19,20}$, a confirmação do declínio fisiológico da mobilidade física ${ }^{20,21}$. Entretanto, não houve diferença significativa nos escores da MIF, o que pode ser explicado pelo fato da MIF ser um instrumento utilizado por meio de entrevista, o que permite o autorrelato e a supervalorização do desempenho. Além disso, dados da literatura mostram que idosos passam a maior parte do dia efetivo executando tarefas necessárias, como as atividades básicas e instrumentais, do que nas demais atividades. Isto pode indicar que mesmo despendendo maior tempo o idoso consegue realizar a tare$\mathrm{fa}^{22}$. Outra justificativa é a inclusão de itens no instrumento relacionados ao desempenho das atividades básicas de vida diária que num gradiente de complexidade estão posicionadas em nível inferior, ou seja, exigem mais competência dos idosos quando comparados a atividades instrumentais e avançadas de vida diária.

Mesmo com a diminuição da mobilidade física, os idosos mais velhos apresentaram melhores pontuações na satisfação com vida, o que corresponde aos achados de outros estudos ${ }^{21,23,24}$. Sato et al. ${ }^{4}$, acrescentaram que esta associação também pode sofrer influencias da combinação de personalidade, cultura e fatores ambientais. 
As estratégias de enfrentamento e a adaptação aos fatos vividos contribuem significativamente para a manutenção da satisfação com a vida através dos anos, mesmo com diminuição da mobilidade física ${ }^{25}$.

Entretanto, alguns estudos trazem relação negativa entre a satisfação com a vida e a funcionalidade, esta não associação pode estar ligada às condições internas do idoso na aceitação do declínio funcional da velhice e de ajuda de outrem nas atividades cotidianas ${ }^{2,26}$.

$\mathrm{O}$ aumento da idade dos idosos do presente estudo favoreceu a 4,77 mais chances de terem melhor pontuação na SV. Isso pode corresponder a existência de certo equilíbrio entre os afetos positivo e negativo vividos pelo idoso. Há uma ênfase na experiência emocional prazerosa e a preponderância de afeto positivo em relação ao negativo, a velhice carrega uma habilidade em trazer lembranças positivas sobre as neutras e estas sobre as lembranças negativas ${ }^{25}$.

As experiências emocionais são vividas com menor intensidade e variedade, frente aos mecanismos de adaptação, que permitem aos idosos poupar recursos já em escassez e aperfeiçoarem a capacidade afetiva e social ${ }^{1,27}$.

Os resultados sugerem que a adaptação dos acontecimentos da vida e as estratégias de enfrentamento dos idosos no decorrer dos anos, são recursos que amenizam a influência do declínio da funcionalidade e mantém o nível de satisfação do bem-estar na velhice ${ }^{1,6,10}$.

O mesmo ocorreu com a SGVC, os sujeitos com 80 anos ou mais apresentaram maior satisfação com vida quando comparada a outra pessoa da mesma idade. Os idosos costumam ava- liar-se em melhores condições satisfatórias ao se compararem com pessoas de mesma idade ${ }^{28}$.

Há evidencia na literatura que essa melhor comparação se dá por mecanismo de enfrentamento denominado de comparação descenden$\mathrm{te}^{29}$. O idoso ao comparar-se com outras pessoas da mesma idade desempenham um papel relevante na avaliação e construção da realidade e enfrentamento de eventos negativos.

As pessoas frequentemente se comparam com outras que elas acreditam ser piores em um esforço de melhorar seu bem-estar, principalmente quando não há oportunidades de ações instrumentais ${ }^{30}$.

Os dados encontrados neste estudo demonstram a forte relação da satisfação com a vida e aumento da idade, também evidenciou a relação com o desempenho físico e a independência funcional.

O conhecimento mais aprofundado da relação entre a SV e a funcionalidade, bem como outros preditores que influenciam a SV, possibilitará o desenvolvimento de métodos para intervir na população idosa, visando o envelhecimento bem sucedido.

Os resultados deste estudo evidenciam o predomínio do sexo feminino bem como maior comprometimento funcional dos idosos mais velhos.

Porém, mesmo com menor desempenho físico, os idosos com 80 anos ou mais, demonstraram maior SV e SVC.

Novos estudos devem ser realizados com abordagem qualitativa que busquem justificativas e melhores detalhamentos sobre a avaliação dos idosos quanto à SV e à SVC. 


\section{Colaboradores}

G Sposito trabalhou na concepção e redação do texto; MJ D'Elboux trabalhou na redação e delineamento do artigo; AL Neri trabalhou na interpretação dos dados e ME Guariento trabalhou na revisão crítica do artigo.

\section{Referências}

1. Neri AL. Qualidade de vida na velhice e subjetividade. In: Neri AL, organizadora. Qualidade de vida na velhice: enfoque multidisciplinar. Campinas: Editora Alínea; 2007. p. 13-59.

2. Diogo, MJD’E. Satisfação global com a vida e determinados domínios entre idosos com amputação de membros inferiores. Rev Panam Salud Publica 2003; 13(6):395-399.

3. Vecchia RD, Ruiz T, Bocchi SCM, Corrente JE. Qualidade de vida na terceira idade: um conceito subjetivo. Rev. Bras Epidemiol 2005; 8(3):246-252.

4. Sato S, Demura S, Kobayashi H, Nagasawa Y. The relationship and its change whit aging betweeen ADL and daily life satisfaction characteristics in independent japanese elderly living at home. J Physiol Anthropol 2002; 21(4):195-204.

5. Neugarten BL. Continuities and discontinuities of psychological issues into adult life: human development. In: Neugarten BL, organizador. Middle age and aging. Chicago: Univerty of Chicago Press; 1969. p. 121-130.

6. Fleck MPA. O instrumento de avaliação de qualidade de vida da Organização Mundial da Saúde (WHOQOL-100): características e perspectivas. Cien Saude Colet 2000; 5(12):33-38.

7. Diener E. Subjective Well Being. Psychological bulletin 1984; 95(3):542-575.

8. Veenhoven R. Happy life expectancy: a comprehensive measure of quality-of-life in nations. Social Indicators Research 1996; 39(1):1-58

9. Bouling A, Gabriel Z, Dukes J, Dowling LM, Evans O, Fleissing A, Banister D, Sutton S. Let's ask them: a national survey of definitions of quality of life and its enhancement among people aged 65 and over. $J$ Aging and Human Development 2003; 56(4):269-306.

10. Diener E, Sush E. Measuring quality of life: economic, social and subjective indicators. Soc Ind Res 1997; 40(1-2):189-216

11. Neri AL. Bienestar subjetivo em la vida adulta y em la vejes: hacia uma psicologia positiva em America Latina. Rev Latinoamericana de Psicologia 2002; 34(12):55-74.

12. Brasil. Ministério da Saúde (MS). Conselho Nacional de Saúde. Resolução no 196 de 10 de outubro de 1996. Diretrizes e Normas Regulamentadoras de Pesquisas Envolvendo Seres Humanos. Diário Oficial da União 1996; 16 out.

13. Guralnik JM, Simonsick EM, Ferrucci L, Glynm RJ, Berkman LF, Blazer DG, Scherr PA, Wallace RB. A Short physical performance battery assessing lower extremity function: association with self-reported disability and prediction of mortality and nursing home admission. J Gerontol 1994; 49(2):85-94.

14. Riberto M, Myazaky MH, Jorge FD, Sakamoto H, Battistela LR. Reprodutividade da versão brasileira da medida de independência funcional. Acta Fisiatr 2001; 8(1):45-52.

15. Hosmer DW, Lemeshow SL. Applied Logistic Regression. New York: Jonh Wiley e Sons; 1989.

16. Chang M, Kim H, Shigematsu R, Nho H, Nishijima T, Tanaka K. Functional fitness may be related to life satisfaction in older Japanese adults. J Aging and Human Development 2001; 53(1):35-49. 
17. Atkinson $\mathrm{HH}$, Cesari M, Kritchevsky SB, Penninx BW, Fried LP, Guralnick JM. Predictors of combined cognitive and physical decline. J Am Geritr Soc 2005; 53(7):1197-1202.

18. Ricci NA, Kubota MT, Cordeiro RC. Concordância de observações sobre a capacidade funcional de idoso em assistência domiciliar. Rev Saude Publica 2005; 39(4):655-662.

19. Barbosa AR, Souza JMP, Lebrão ML, Laurenti R, Marucci MFN. Functional limitations of Brazilian elderly by age and gender differences. SABE Survey 2005; 21(4):1177-1185.

20. Mollaoglu M, Tuncay FO, Fertelli TK. Mobility disability and life satisfaction in elderly people. Arch Gerontol Geriatr 2010; 51(3):115-119

21. Meléndez JC, Tomás JM, Oliver A, Navarro E. Psychological and physical dimensions explaining life satisfaction among the elderly: A structural model examination. Arch Gerontol Geriatr 2009; 48(3):291-295.

22. Doimo LA, Derntl AM, Lago OC. O uso do tempo no cotidiano de mulheres idosas: um método indicador do estilo de vida de grupos populacionais. Cien Saude Colet 2008; 13(4):1133-1142.

23. Enkvist A, Ekstron H, Elmstahl S. What factors affect life satisfaction (LS) among the oldest-old? Arch Gerontol Geriatr 2012; 54(1):140-145.

24. Mhaoláin AMN, Gallagher D, O Connell H, Chin AV, Bruce I, Hamilton F, Teehee E, Coen R, Coakley D, Cunningham C, Walsh JB, Lawlor BA. Subjective well-being amost community-dwelling elders: what determines satisfaction whit life? Findings from the Dublin Healthy Aging Study. International Psycho Int Psychogeriatr 2012; 24(2):316-323.

25. Diener E, Suh ME. Subjetive well-being and age: An international analysis. In: Schaie KW, Lawton MP, editors. Annual Review of Gerontology and Geriatrics. New York: Springer Publishing Company; 2003. p. 305-324.
26. Hernadez R, Fernádez L, Alonso O. Satisfaction with life to functionality in active elderly people. Acts Esp Psiquiatr 2009; 37(2):61-67.

27. Carstensen LL. Motivation for social contact across the life span: a theory of socioemotional selectivy. In: Jacobs JE, organizador. Nebraska Symposium on motivation, 1002: developmental perspectives on motivation. Lincoln: University of Nebraska Press; 1993. p. 209-254.

28. Diener E, Larsen R. The experience of emotional well-being. In: Lewis M, Haviland JM, organizador. Handbook of emotions. Ney York: Guildford; 1993. p. 405-415.

29. Wills TA. Modes and Families of coping: an analysis of of social comparision in the structure of other cognitive and behavioral mechanisms. In: Buunk BP, Gibbsons F, organizadores. Helath, coping, and well-being: perspective from social comparison theory. Mahwal: Laurence Erlbawm Associates; 1997. p. 167194.

30. Buunk BP, Gibbons FX, Reis-Bergan M. Social comparision in health and illness: a historical overview. In: Buunk BP, Gibbsons F, organizadores. Health, coping, and well-being: perspective from social comparison theory. Mahwal: Laurence Erlbawm Associates; 1997. p. 1-23.

Artigo apresentado em 22/09/2012

Aprovado em 30/10/2012

Versão final apresentada em 05/11/2012 